\title{
EL MÉTODO DE DESCOMPOSICIÓN DE ADOMIAN PARA EL CÁLCULO DE INTEGRALES GAUSSIANAS: ECUACIÓN DE CALOR Y DE BLACK-SCHOLES
}

\author{
OSWALDO GONZÁLEZ-GAXIOLA
}

\begin{abstract}
Resumen. Derivada por los economistas Fischer Black, Myron Scholes y Robert Merton en 1973, la ecuación de Black-Scholes es una ecuación diferencial parcial cuya solución es utilizada para determinar cuánto vale en un momento dado una opción de compra o venta en principio tipo europea. En el presente trabajo proponemos una adaptación del método de descomposición de Adomian, que permite la evaluación de ciertas integrales gaussianas en términos de una serie convergente. Además haremos la aplicación del método expuesto para encontrar soluciones a la ecuación de Black-Scholes bajo cierta restricción impuesta por la condición inicial.
\end{abstract}

\section{INTRODUCCIÓN}

La mayor parte de los fenómenos que surgen en el mundo real son descritos por ecuaciones diferenciales no lineales (tanto ordinarias como parciales) y en algunos caso por ecuaciones integrales. Sin embargo, los métodos más comunes desarrollados hasta ahora en matemáticas se utilizan para resolver ecuaciones diferenciales lineales. En la década de los años 80, George Adomian introdujo un nuevo método para resolver ecuaciones diferenciales no lineales, tanto parciales como ordinarias e incluso estocásticas 1, 2, 3. Este método ha sido denominado como el método de descomposición de Adomian (ADM). Se ha demostrado que el método de descomposición resuelve de manera eficiente, fácil y precisa una gran clase de ecuaciones diferenciales lineales y no lineales ordinarias, parciales, integrales, de orden entero o fraccional, deterministas o estocásticas [4, 5, 6, 7, 8, El método se adapta muy bien tanto a problemas físicos como de otras áreas del conocimiento, ya que no requiere de linealizaciones, perturbaciones y otras suposiciones restrictivas que pueden cambiar la naturaleza del problema que se está modelando y resolviendo. ADM tiene la ventaja de que converge a la solución exacta en una gran mayoría de casos importantes en las aplicaciones y se puede manejar de manera relativamente fácil pues el método genera a través de su algoritmo una solución en forma de una serie cuyos términos son determinados por una relación recursiva. Algunos trabajos fundamentales sobre diversos aspectos de las modificaciones de ADM asi como el estudio de la convergencia se pueden ver en [9, 10, 11, 12, 13].

Por otra parte, en 1973, Robert C. Merton, junto a Fisher Black y Myron Scholes desarrolló el modelo de Black-Scholes. Merton ayudo a introducir el cálculo estocástico en la economía financiera, lo que permitió que el comportamiento de los precios fuese descrito con el lenguaje preciso de la probabilidad. Al modelo obtenido Robert Merton denominó la ecuación de Black-Scholes [14. En 1997 el Premio Nobel de Economía fue para Robert C. Merton y Myron S. Scholes, por contribuir a las ciencias económicas, con un nuevo método para determinar el valor de los derivados, antes Fisher Black falleció y por lo tanto no pudo recibir este premio.

En el presente trabajo haremos una adaptación de la versión estándar de ADM para calcular integrales gaussianas y así poder hallar la solución de la ecuación de calor y

2010 Mathematics Subject Classification. 35K05, 35Q91 91G15.

Palabras clave. Método de descomposición de Adomian, ecuación de Black-Scholes, integrales gaussianas. 
de ello se derivará una alternativa de solución para la ecuación de Black-Scholes en su versión lineal.

\section{Una Breve Descripción del Método de Descomposición de Adomian}

El método que a continuación expondremos es un método poderoso para resolver ecuaciones diferenciales no lineales. El método se basa en descomponer la solución de una ecuación diferencial lineal o no lineal en una serie de funciones, cada término de esta serie se obtiene de manera polinomial y recursiva partiendo de una condición inicial que requiere ser una función analítica, la técnica es muy simple en una formulación abstracta pero la dificultad surge al calcular cada uno de los polinomios especialmente en los modelos descritos por ecuaciones diferenciales no lineales.

Para establecer la idea básica de una manera sencilla, consideramos la ecuación diferencial ya sea ordinaria o parcial, en general no lineal, en la forma [15]:

$$
F u(x, t)=g(x, t)
$$

con la condición inicial

$$
u(x, 0)=f(x),
$$

donde $F$ representa un operador diferencial (en general, no lineal) que envuelve tanto términos lineales como no lineales y entonces la ecuación (1) puedes escribirse como

$$
L_{t} u(x, t)+R u(x, t)+N u(x, t)=g(x, t)
$$

donde $L_{t}=\frac{\partial}{\partial t}, R$ es un operador lineal que involucra derivadas parciales con respecto a $x$ y $N$ es un operador no lineal; $g$ es un término no homogéneo independiente de $u$. Despejando $L_{t} u(x, t)$,

$$
L_{t} u(x, t)=g(x, t)-R u(x, t)-N u(x, t)
$$

Como $L$ es invertible, operando en $\sqrt{4}$ con el inverso $L_{t}^{-1}(\cdot)=\int_{0}^{t}(\cdot) d z$ tenemos que,

$$
L_{t}^{-1} L_{t} u(x, t)=L_{t}^{-1} g(x, t)-L_{t}^{-1} R u(x, t)-L_{t}^{-1} N u(x, t)
$$

luego, una expresión equivalente a (5) es

$$
u(x, t)=f(x)+L_{t}^{-1} g(x, t)-L_{t}^{-1} R u(x, t)-L_{t}^{-1} N u(x, t)
$$

donde $f(x)$ es la constante de integración (con respecto a $t$ ) que satisface $L_{t} f=0$. Para problemas con valor inicial en $t=t_{0}$, tendríamos convenientemente definido $L^{-1}$ para $L=\frac{\partial^{n}}{\partial t^{n}}$ como la integral $n$-veces iterada definida de $t_{0}$ a $t$.

Por el método (ADM) asumimos la solución de (1), 22 en forma de serie para la función desconocida $u(x, t)$ dada por,

$$
u(x, t)=\sum_{n=0}^{\infty} u_{n}(x, t)
$$

El término no lineal $N u(x, t)$ por medio de ADM se descompone como

$$
N u(x, t)=\sum_{n=0}^{\infty} A_{n}\left(u_{0}, u_{1}, \ldots, u_{n}\right)
$$

donde la sucesión $\left\{A_{n}\right\}_{n=0}^{\infty}$, es la llamada sucesión de polinomios de Adomian y son dados por la fórmula

$$
A_{n}=\left.\frac{1}{n !} \frac{d^{n}}{d \alpha^{n}}\left[N\left(\sum_{k=0}^{n} \alpha^{k} u_{k}\right)\right]\right|_{\alpha=0} .
$$

En [16] podemos ver que el cálculo explícito de cada uno de los $A_{n}$ es realmente sencillo y se tiene:

$$
\begin{aligned}
& A_{0}\left(u_{0}\right)=N\left(u_{0}\right) \\
& A_{1}\left(u_{0}, u_{1}\right)=N^{\prime}\left(u_{0}\right) u_{1} \\
& A_{2}\left(u_{0}, u_{1}, u_{2}\right)=N^{\prime}\left(u_{0}\right) u_{2}+\frac{u_{1}^{2}}{2 !} N^{\prime \prime}\left(u_{0}\right)
\end{aligned}
$$




$$
\begin{aligned}
& A_{3}\left(u_{0}, \ldots, u_{3}\right)=N^{\prime}\left(u_{0}\right) u_{3}+N^{\prime \prime}\left(u_{0}\right) u_{1} u_{2}+\frac{u_{1}^{3}}{3 !} N^{\prime \prime \prime}\left(u_{0}\right) \\
& A_{4}\left(u_{0}, \ldots, u_{4}\right)=u_{4} N^{\prime}\left(u_{0}\right)+\left(\frac{1}{2 !} u_{2}^{2}+u_{1} u_{3}\right) N^{\prime \prime}\left(u_{0}\right)+\frac{u_{1}^{2} u_{2}}{2 !} N^{\prime \prime \prime}\left(u_{0}\right)+\frac{u_{1}^{4}}{4 !} N^{(i v)}\left(u_{0}\right)
\end{aligned}
$$

$\vdots$

Ahora, sustituyendo (7), (8) y (9) en la ecuación (6) obtenemos,

$$
\sum_{n=0}^{\infty} u_{n}(x, t)=f(x)+L_{t}^{-1} g(x, t)-L_{t}^{-1} R \sum_{n=0}^{\infty} u_{n}(x, t)-L_{t}^{-1} \sum_{n=0}^{\infty} A_{n}\left(u_{0}, u_{1}, \ldots, u_{n}\right),
$$

de donde obtenemos el algoritmo recursivo

$$
\left\{\begin{array}{l}
u_{0}(x, t)=f(x)+L_{t}^{-1} g(x, t), \\
u_{n+1}(x, t)=-L_{t}^{-1} R u_{n}(x, t)-L_{t}^{-1} A_{n}\left(u_{0}, u_{1}, \ldots, u_{n}\right), n=0,1,2, \ldots
\end{array}\right.
$$

Con el algoritmo recursivo establecido en la ecuación (11), la aproximación a $n-$ términos de la solución de (1)-2) la obtenemos como

$$
S_{k}(x, t)=\sum_{n=0}^{k} u_{n}(x, t) \quad \text { con } u(x, t)=\lim _{k \rightarrow \infty} S_{k}(x, t)
$$

Como sabemos, cuantos más términos se agreguen a la solución aproximada, más precisa será.

La descomposición de la solución en serie, generalmente converge muy rápido. Las condiciones para las cuales el método converge no son objetivo del presente estudio y han sido estudiadas principalmente en los trabajos [10], 11], 12] y [13] así como en varias de sus referencias.

\section{La ECuación de CALOR y ADM}

Ahora veremos que ADM resulta ser un procedimiento efectivo para la evaluación de ciertas integrales que presentan cierto grado de dificultad como son las integrales gaussianas. Para abordar este problema, comenzamos por considerar la ecuación de calor para una varilla infinita con una condición inicial arbitraria:

$$
\left\{\begin{array}{l}
\frac{\partial u}{\partial t}=k \frac{\partial^{2} u}{\partial x^{2}}, x \in \mathbb{R}, t>0, k>0 \\
u(x, 0)=f(x) \\
|u(x, t)| \leq M \text { para toda } x \in \mathbb{R} \text { y } t>0
\end{array}\right.
$$

La representación explícita de la solución al problema de valor inicial 13 es dada por

$$
u(x, t)=\frac{1}{\sqrt{4 k \pi t}} \int_{-\infty}^{\infty} f(\xi) e^{-\frac{(\xi-x)^{2}}{4 k t}} d \xi, \quad-\infty<x<\infty, t>0 .
$$

En el integrando de 14 la función $G(x, t)=\frac{1}{\sqrt{4 k \pi t}} e^{-\frac{x^{2}}{4 k t}} d \xi$ es conocida como la solución fundamental de la ecuación de calor. Para mayor referencia al respecto ver [17.

Puede suceder que la integral en el lado derecho de (14) no sea expresable en términos de funciones elementales y menos aún pueda ser calculada de manera exacta por medio de los métodos elementales del cálculo integral. En el presente trabajo buscamos evaluar este tipo de integrales que involucran la función de error usando el método de descomposición de Adomian. El presente trabajo no es el primero en abordar el problema de evaluar integrales a través de un método de descomposición, para mayor información podemos ver [18, 19] y sus referencias.

Consideremos el problema de valor inicial dado por la ecuación de calor 13 . Primero, definimos los operadores lineales $L$ y $R$ en el contexto de ADM como

$$
L_{t}=\frac{\partial}{\partial t} \quad \text { y } R=\frac{\partial^{2}}{\partial x^{2}}
$$


Con esto podemos escribir la ecuación diferencial parcial de 13 como

$$
L_{t} u=k R u \text {. }
$$

Ahora vamos a considerar el operador inverso de $L_{t}$, esto es, $L_{t}^{-1}(u)=\int_{0}^{t} \frac{\partial u}{\partial \xi} d \xi$ para aplicarlo a ambos lados de (16) para obtener, usando el teorema fundamental del cálculo y la condición inicial $f(x)=u(x, 0)$ :

$$
u(x, t)=f(x)+k L_{t}^{-1} R u .
$$

Haciendo uso de (7), descomponemos la solución en serie de componentes $u_{n}$, esto es,

$$
u(x, t)=\sum_{n=0}^{\infty} u_{n}(x, t) .
$$

Sustituyendo (18) en (17), obtenemos

$$
\sum_{n=0}^{\infty} u_{n}(x, t)=f(x)+k \int_{0}^{t} \sum_{n=0}^{\infty} \frac{\partial^{2} u_{n}}{\partial x^{2}}(x, \xi) d \xi .
$$

Finalmente, considerando (19) e igualando sumandos del mismo orden obtenemos el algoritmo para encontrar las componentes de la serie (18):

$$
\left\{\begin{array}{l}
u_{0}(x, t)=f(x) \\
u_{n+1}(x, t)=k \int_{0}^{t} \frac{\partial^{2} u_{n}}{\partial x^{2}}(x, \xi) d \xi, n=0,1,2, \ldots
\end{array}\right.
$$

Consecuentemente, tenemos

$$
\begin{aligned}
u_{0}(x, t) & =f(x), \\
u_{1}(x, t) & =k f^{(2)}(x) t, \\
u_{2}(x, t) & =k^{2} f^{(4)}(x) \frac{t^{2}}{2 !}, \\
& \vdots \\
u_{n}(x, t) & =k^{n} f^{(2 n)}(x) \frac{t^{n}}{n !}
\end{aligned}
$$

Del esquema anterior la solución de la ecuación de calor es dada por

$$
u(x, t)=\sum_{n=0}^{\infty} k^{n} f^{(2 n)}(x) \frac{t^{n}}{n !},
$$

donde $f^{(j)}(x)$ significa la $j$-ésima derivada de $f$ respecto a $x$.

Como resumen de todo lo anterior, podemos establecer el siguiente teorema.

Teorema 1. Sea $f: \mathbb{R} \rightarrow \mathbb{R}$ una función $C^{\infty}(\mathbb{R})$. Entonces para $-\infty<x<\infty$ se tiene

$$
\int_{-\infty}^{\infty} f(\xi) e^{-\frac{(\xi-x)^{2}}{4 k t}} d \xi=\sqrt{4 k \pi t} \sum_{n=0}^{\infty} f^{(2 n)}(x) \frac{(k t)^{n}}{n !}, \quad t>0, \quad k>0 .
$$

Demostración. La demostración se sigue directamente de utilizando 21) y 14 .

Corolario 2. Sea $f: \mathbb{R} \rightarrow \mathbb{R}$ una función $C^{\infty}(\mathbb{R})$. Entonces para $b>0$ se tiene

$$
\int_{-\infty}^{\infty} f(x) e^{-b x^{2}} d x=\sqrt{\frac{\pi}{b}} \sum_{n=0}^{\infty} \frac{f^{(2 n)}(0)}{4^{n} b^{n} n !} .
$$

La fórmula (23) nos proporciona una herramienta útil para calcular integrales gaussianas en las cuales la función $f$ cumplan con la condición de diferenciabilidad. 


\section{Aplicación a la Ecuación de Black-Scholes}

La ecuación de Black-Scholes es una ecuación diferencial parcial de segundo orden de tipo parabólico utilizada en matemáticas financieras modernas para determinar el precio de activos financieros, y esta es dada por [14, 20]:

$$
V_{t}+\frac{1}{2} \sigma^{2} S^{2} V_{S S}+(r-\delta) S V_{S}-r V=0,
$$

la ecuación 24 es una ecuación diferencial parcial parabólica definida en

$$
D_{V}=\{(S, t): S>0,0<t \leq T\} .
$$

En la ecuación de Black-Scholes (24) tenemos que $V=V(S, t)$ es el precio de una opción o también conocida como función de pago, $S=S(t)$ representa el precio de un activo subyacente al tiempo $t, r$ es la tasa de interés del mercado de deuda libre de riesgo, $\delta$ representa los dividendos que se reparten de forma continua y $\sigma$ es la volatilidad de la acción, medida como la desviación estándar de los logaritmos de la cotización de la acción y que aquí supondremos constante.

El modelo dado por (24) tiene varias suposiciones técnicas y propias del lenguaje financiero, las principales son que $r$, es conocida y constante en el tiempo, no hay costos de transacción en la compra o venta del activo por la opción, no hay impuestos $\mathrm{u}$ otras tasaciones y no existen oportunidades de arbitraje sin riesgo.

Siguiendo la idea dada en [21, consideremos los siguientes cambios de variables; para las variables independientes

$$
S=e^{x}, \quad t=T-\frac{2 \tau}{\sigma^{2}}
$$

y para la variable dependiente

$$
v(x, \tau)=V(S, t)=V\left(e^{x}, T-\frac{2 \tau}{\sigma^{2}}\right) .
$$

Ahora, calculando tenemos:

$$
\begin{gathered}
\frac{\partial V}{\partial t}=\frac{\partial v}{\partial \tau} \frac{\partial \tau}{\partial t}=-\frac{\sigma^{2}}{2} \frac{\partial v}{\partial \tau} \\
\frac{\partial V}{\partial S}=\frac{\partial v}{\partial x} \frac{\partial x}{\partial S}=\frac{1}{S} \frac{\partial v}{\partial x} \\
\frac{\partial^{2} V}{\partial S^{2}}=\frac{\partial}{\partial S}\left(\frac{\partial V}{\partial S}\right)=\frac{1}{S^{2}}\left[\frac{\partial^{2} v}{\partial x^{2}}-\frac{\partial v}{\partial x}\right] .
\end{gathered}
$$

Sustituyendo en (24) obtenemos

$$
v_{\tau}=v_{x x}+\left(\frac{r-\delta}{\sigma^{2} / 2}-1\right) v_{x}-\frac{r}{\sigma^{2} / 2} v
$$

Ahora definimos los coeficientes como

$$
\kappa=\frac{r-\delta}{\sigma^{2} / 2}, \quad l=\frac{\delta}{\sigma^{2} / 2},
$$

de donde podemos reescribir la ecuación (25) como

$$
v_{\tau}=v_{x x}+(\kappa-1) v_{x}-(\kappa+l) v .
$$

La ecuación $(26)$ tiene coeficientes constantes.

Además proponemos el siguiente cambio de variable dependiente

$$
v(x, \tau)=e^{-\gamma x-\left(\beta^{2}+l\right) \tau} u(x, \tau),
$$

donde

Derivando obtenemos

$$
\gamma=\frac{1}{2}(\kappa-1), \quad \text { y } \beta=\frac{1}{2}(\kappa+1) \text { y por lo tanto } \beta^{2}=\gamma^{2}+\kappa .
$$

$$
v_{\tau}=e^{-\gamma x-\left(\beta^{2}+l\right) \tau}\left(u_{\tau}-\left(\beta^{2}+l\right) u\right),
$$




$$
v_{x}=e^{-\gamma x-\left(\beta^{2}+l\right) \tau}\left(u_{x}-\gamma u\right), \quad v_{x x}=e^{-\gamma x-\left(\beta^{2}+l\right) \tau}\left(u_{x x}+\gamma^{2} u-2 \gamma u_{x}\right) .
$$

Sustituyendo en la ecuación 26.

$$
u_{\tau}=u_{x x}+(\kappa-1-2 \gamma) u_{x}+\gamma(2 \gamma-\kappa+1) u=u_{x x} .
$$

Por lo tanto, la ecuación que satisface la variable dependiente $u(x, \tau)$ es la forma adimensional de la ecuación de calor:

$$
\frac{\partial u}{\partial t}=\frac{\partial^{2} u}{\partial x^{2}} .
$$

Como consecuencia de lo anterior, la ecuación de Black-Scholes (24) es equivalente a la ecuación de calor definida en

$$
D_{u}=\left\{(x, \tau):-\infty<x<\infty, 0 \leq \tau \leq \frac{\sigma^{2}}{2} T\right\} .
$$

En general, la ecuación de calor está definida para todo $\tau \geq 0$ pero considerando que una opción expira al tiempo de madurez $t=T$ el dominio de definición es el conjunto $D_{u}$.

Debido al Teorema 1 si se conoce la condición inicial suficientemente suave $u(x, 0)=$ $g(x)$, entonces la solución de la ecuación de Black-Scholes es dada por

$$
u(x, \tau)=\sqrt{4 \pi \tau} \sum_{n=0}^{\infty} g^{(2 n)}(x) \frac{\tau^{n}}{n !}, \quad \tau \geq 0 .
$$

Finalmente, no debemos olvidar que la relación entre las variables originales $(S, t)$ en $D_{V}$ y las transformadas $(x, \tau)$ en $D_{u}$ esta dada por:

$$
x=\ln (S), \quad \tau=\frac{\sigma^{2}}{2}(T-t) .
$$

Cabe aclarar que la solución que hemos obtenido a partir del método de descomposición expuesto tiene como limitante el requisito de una condición inicial con alto grado de diferenciabilidad. Además, los mercados del mundo real tienen comportamientos no lineales [22, 23], un modelo con volatilidad no constante que resulta modificada por el mercado financiero tiene diferentes tratamientos y muchos de ellos matemáticamente muy sofisticados. Al verse modificada la volatilidad el modelo afecta el costo de las transacciones y se presenta iliquidez financiera, entre otras posibles afectaciones. En 24] presentamos una versión no lineal del modelo de Black-Scholes derivada de los costos de transacción así como también de la iliquidez del mercado, y la solución de dicho modelo a través del mismo método aquí empleado, en él se considera la volatilidad como una función que depende del tiempo, del precio del activo subyacente y de la prima de la opción.

\section{COnClusiones}

En el presente trabajo hemos expuesto de manera breve el método de descomposición estándar de Adomian (ADM), también basados en el método y en la ecuación de difusión del calor hemos obtenido una manera práctica de evaluar integrales gaussianas a través de la serie proporcionada por ADM sin tener que recurrir a discretizaciones, perturbaciones u otras suposiciones que en ocasiones distorsionan la naturaleza del problema. Además, hemos dado una breve introducción al modelo matemático establecido por la ecuación de Black-Scholes en su versión lineal, la cual puede transformarse en una ecuación de difusión y dependiendo de la condición inicial puede ser resuelta por medio del método estudiado. Finalmente, podemos concluir que el método que hemos mostrado es una herramienta poderosa y no sofisticada que puede utilzarse además de para el cálculo de integrales gaussianas y resolver algunas ecuaciones diferenciales que surgen en las aplicaciones de las matemáticas. 


\section{REFERENCIAS}

[1] Adomian, G., Solving Frontier Problems of Physics: The Decomposition Method, Boston, MA: Kluwer Academic Publishers, 1994.

[2] Adomian, G. A review of the decomposition method in applied mathematics. J. Math. Anal. Appl., 135, No. 2, 501-544, 1988.

[3] Adomian, G., Nonlinear stochastic operator equations, New York, Academic Press, 1986.

[4] Gejji, V. D. \& Jafari, H. Adomian decomposition: a tool for solving a system of fractional differential equations. J. Math. Anal. Appl., 301, 508-518, 2005.

[5] Khuri, S. A. A Laplace decomposition algorithm applied to class of nonlinear differential equations. J. Math. Appl., 4, 141-155, 2001.

[6] Khuri, S. A. A new approach to Bratu's problem. Appl. Math. Comput., 147, 131-136, 2004.

[7] González-Gaxiola, O. \& Biswas, A. Akhmediev breathers, Peregrine solitons and KuznetsovMa solitons in optical fibers and PCF by Laplace-Adomian decomposition method. Optik, 172, 930-939, 2018.

[8] Adomian, G. \& Serrano, S. E. Stochastic contaminant transport equation in porous media. Appl. Math. Lett., 11, 53-55, 1998.

[9] Venkatarangan, S. N. \& Rajalakshmi, K. A modification of Adomian?s solution for nonlinear oscillatory systems. Comput. Math. Appl., 29, 67-73, 1995.

[10] Cherruault, Y. Convergence of Adomian's method. Kybernetes, 18, No. 2, 31-38, 1989.

[11] Cherruault, Y. \& G. Adomian, G. Decomposition methods: a new proof of convergence. Math. Comput. Modelling, 18, No. 12, 103-106, 1993.

[12] Abbaoui, K. \& Cherruault Y. Convergence of Adomian's method applied to differential equations. Comput. Math. Appl., 28, No. 5, 103-109, 1994.

[13] Abbaoui, K. \& Cherruault Y. New ideas for proving convergence of decomposition methods. Comput. Math. Appl., 29, No. 7, 103-108, 1995.

[14] Black,F. \& Scholes, M. The pricing of option and corporate liabilities. The Journal of Political Economy, 81, No. 3, 637-654, 1973.

[15] Wazwaz, A. M., Partial Differential Equations: Methods and Applications, Lisse, Netherlands: Balkema Publishers, 2002.

[16] Wazwaz, A. M. A new algorithm for calculating adomian polynomials for nonlinear operators. Appl. Math. and Computation, 111, No. 1, 53-69, 2000.

[17] Strauss, W. A., Partial Differential Equations: An Introduction, John Wiley and Sons, Inc., 1992.

[18] Adomian, G. \& Rach, R. Evaluation of integrals by decomposition. J. of Comput. and Applied Math., 23, 99-101, 1986.

[19] Sandoval-Hernández, M. A., Vazquez-Leal, H., Filobello-Nino, U. \& Hernández-Martínez, L. New handy and accurate approximation for the Gaussian integrals with applications to science and engineering. Open Math., 17, 1774-1793, 2019.

[20] Cox, J. C., Ross, S. A. \& Rubinstein, M. Option pricing: A simplified approach. J. of Financial Economics, 7, 229-263, 1979.

[21] Gal, C. G., Gal, S. G. \& Goldstein, J. A. Burgers and Black-Merton-Scholes equations with real time variable and complex spatial variable. Applicable Analysis, 92, No. 8, 1766-1786, 2013.

[22] Frey, R. \& Stremme, A. Market volatility and feedback effects from dynamic hedging. Math. Financ., 4, 351-374, 1997.

[23] Liu, H. \& Yong, J. Option pricing with an illiquid underlying asset market. J. Econ. Dyn. Control, 29, 2125-2156, 2005.

[24] González-Gaxiola, O., Ruiz de Chávez J. \& Santiago, J. A. A nonlinear option pricing model through the Adomian decomposition method. Int. J. Appl. Comput. Math., 2, 453-467, 2016.

\section{Dirección del autor:}

Universidad Autónoma Metropolitana,

Unidad Cuajimalpa,

División de Ciencias Naturales e Ingeniería,

Departamento de Matemáticas Aplicadas y Sistemas.

Av. Vasco de Quiroga 4871, Santa Fe, Cuajimalpa

C.P. 05300, Ciudad de México.

e-mail: ogonzalez@cua.uam.mx 